\title{
Article
}

\section{Sparkling extreme-ultraviolet bright dots observed with Hi-C}

Regnier, Stephane, Alexander, Caroline, Walsh, Robert William, Winebarger, A. R., Cirtain, J., Golub, L., Korreck, K. E., Mitchell, Nicholas Philip, Platt, Simon Philip, Weber, M, De Pontieu, B, Title, A, Kobayashi, K, Kuzin, S and DeForest, C E

Available at https://clok.uclan.ac.uk/10930/

Regnier, Stephane orcid iconORCID: 0000-0001-8954-4183, Alexander, Caroline, Walsh, Robert William orcid iconORCID: 0000-0002-1025-9863,

Winebarger, A. R., Cirtain, J., Golub, L., Korreck, K. E., Mitchell, Nicholas Philip, Platt, Simon Philip orcid iconORCID: 0000-0003-4431-8814 et al (2014) Sparkling extreme-ultraviolet bright dots observed with Hi-C. The Astrophysical Journal, 784 (2). p. 134. ISSN 0004-637X

It is advisable to refer to the publisher's version if you intend to cite from the work. http://dx.doi.org/10.1088/0004-637x/784/2/134

For more information about UCLan's research in this area go to http://www.uclan.ac.uk/researchgroups/ and search for <name of research Group>.

For information about Research generally at UCLan please go to http://www.uclan.ac.uk/research/

All outputs in CLoK are protected by Intellectual Property Rights law, including Copyright law. Copyright, IPR and Moral Rights for the works on this site are retained by the individual authors and/or other copyright owners. Terms and conditions for use of this material are defined in the policies page.

\section{CLoK}

Central Lancashire online Knowledge www.clok.uclan.ac.uk

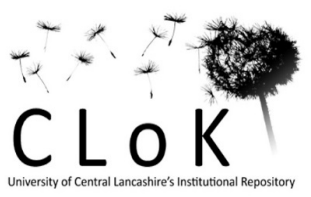




\title{
SPARKLING EXTREME-ULTRAVIOLET BRIGHT DOTS OBSERVED WITH Hi-C
}

\author{
S. Régnier ${ }^{1}$, C. E. Alexander ${ }^{1}$, R. W. Walsh ${ }^{1}$, A. R. Winebarger ${ }^{2}$, J. Cirtain ${ }^{2}$, L. Golub ${ }^{3}$, \\ K. E. Korreck ${ }^{3}$, N. Mitchell ${ }^{4}$, S. Platt ${ }^{4}$, M. Weber ${ }^{3}$, B. De Pontied ${ }^{5}$, A. Title ${ }^{5}$, \\ K. Kobayashi ${ }^{6}$, S. KuZIN ${ }^{7}$, And C. E. DeForest ${ }^{8}$ \\ ${ }^{1}$ Jeremiah Horrocks Institute, University of Central Lancashire, Preston, Lancashire, PR1 2HE, UK \\ ${ }^{2}$ NASA Marshall Space Flight Center, VP 62, Huntsville, AL 35812, USA \\ ${ }^{3}$ Harvard-Smithsonian Center for Astrophysics, 60 Garden Street, Cambridge, MA 02138, USA \\ ${ }^{4}$ School of Computing, Engineering and Physical Sciences, University of Central Lancashire, Preston, Lancashire, PR1 2HE, UK \\ ${ }^{5}$ Lockheed Martin Solar and Astrophysics Laboratory, 3251 Hanover Street, Palo Alto, CA 94304, USA \\ ${ }^{6}$ Center for Space Plasma and Aeronomic Research, 320 Sparkman Dr, Huntsville, AL 35805, USA \\ ${ }^{7}$ Lebedev Physical Institute, Russian Academy of Sciences, Leninskii pr. 53, Moscow, 119991, Russia \\ ${ }^{8}$ Southwest Research Institute, 1050 Walnut Street Suite 300, Boulder, CO 80302, USA \\ Received 2013 September 17; accepted 2014 February 7; published 2014 March 14
}

\begin{abstract}
Observing the Sun at high time and spatial scales is a step toward understanding the finest and fundamental scales of heating events in the solar corona. The high-resolution coronal (Hi-C) instrument has provided the highest spatial and temporal resolution images of the solar corona in the EUV wavelength range to date. Hi-C observed an active region on 2012 July 11 that exhibits several interesting features in the EUV line at $193 \AA$. One of them is the existence of short, small brightenings "sparkling" at the edge of the active region; we call these EUV bright dots (EBDs). Individual EBDs have a characteristic duration of $25 \mathrm{~s}$ with a characteristic length of $680 \mathrm{~km}$. These brightenings are not fully resolved by the SDO/AIA instrument at the same wavelength; however, they can be identified with respect to the Hi-C location of the EBDs. In addition, EBDs are seen in other chromospheric/coronal channels of $S D O /$ AIA, which suggests a temperature between 0.5 and $1.5 \mathrm{MK}$. Based on their frequency in the Hi-C time series, we define four different categories of EBDs: single peak, double peak, long duration, and bursty. Based on a potential field extrapolation from an $S D O / \mathrm{HMI}$ magnetogram, the EBDs appear at the footpoints of large-scale, trans-equatorial coronal loops. The Hi-C observations provide the first evidence of small-scale EUV heating events at the base of these coronal loops, which have a free magnetic energy of the order of $10^{26} \mathrm{erg}$.
\end{abstract}

Key words: Sun: activity - Sun: corona - Sun: magnetic fields - Sun: UV radiation

Online-only material: color figures

\section{INTRODUCTION}

A long-standing problem in solar physics is how the solar corona is heated up to a temperature of a few million degrees while the photosphere is at about $6000 \mathrm{~K}$. Several models have been developed that either heat the coronal plasma locally or transport heat from the lower layers of the solar atmosphere into the corona (see, for instance, the review by Klimchuk 2006); however, these models often lack an explanation for an average temperature of $1 \mathrm{MK}$ for the entire corona and its sustainability during a solar cycle. This strongly suggests that several mechanisms may be at play, each of them providing a substantial amount of heat to the corona, and can vary during the activity cycle of the Sun. Those models also invoke smaller spatial and timescales than the ones provided by the current observations.

So, the critical issue is really to how to answer the questions: Where is the source of heat located, and where and how does the heating take place? Along a given magnetic structure (often a coronal loop), three scenarios are often considered: the release of energy is located (1) at the footpoints of the structure in the photospheric layer, which is the most favorable location thanks to the importance of the photospheric motions and the source of material and energy transported from the convection zone, (2) at the apex of the structure, and (3) uniformly along the structure. Those three scenarios imply that the heat is transported along the magnetic structures, and that we actually observe loop-like structures in the corona. In terms of observations, it has been difficult to disentangle the actual location of the heat source. As an example, several authors using a data set obtained in soft X-rays by Yohkoh/SXT have reached an opposite conclusion depending on how they dealt with the data reduction (Mackay et al. 2000; Priest et al. 2000; Reale 2002). To determine the possible heating scenario, the authors looked at the profile of the temperature along the coronal loop and compared that with a theoretical model. These indirect methods were necessary because of the coarse spatial and time resolutions of Yohkoh/SXT (compared to what can be achieved today). Thanks to the development of new high spatial and time resolution instrumentation, in this paper we are looking for direct evidence of the source of heat deposition, which is observationally characterized by a small, short increase in intensity.

Theoretically, the different heating locations (apex, uniform, or footpoint) arise from the idea that magnetic energy stored in the chromosphere/corona produces small, short bursts converting the magnetic energy into thermal and/or kinetic energy (rapidly converted into heat). This is the basic principle of Parker's theory (1988) for heating the solar corona by nanoflares. A large number of those small events is needed to sustain and uniformly distribute the heat in the entire solar corona. The mechanism responsible for the release of magnetic energy could be, for instance, magnetic reconnection, wave mode coupling, or turbulence. As a large amount of the free energy contained in the magnetic field structure of active regions is located at the base of the corona or in the chromosphere (Régnier \& Priest 
2007), the most favorable heating location is at the footpoints of coronal loops. For the quiet Sun, the footpoints of the coronal structures are most affected by the photospheric motions, such as granular motions that can easily produce tangled, braided, or twisted magnetic flux bundles. In this paper, we combine EUV observations and a magnetic field model to determine if Parker's model is a viable scenario, even if we cannot identify the mechanism(s) responsible for the energy release.

The high-resolution, ground-based instruments in the visiblewavelength domain allowed observations of new structures and phenomena in the lower layers of the solar atmosphere, especially small-scale brightenings and events such as spicules or Ellerman bombs (see, e.g., Scharmer et al. 2003; Berger et al. 2004; Rutten et al. 2004; Rouppe van der Voort et al. 2005; Beck et al. 2007). In conjunction, the investigation of the coronal plasma at EUV wavelengths is being constantly improved in terms of spatial and temporal resolution. For instance, the Atmospheric Imager Assembly (AIA; Lemen et al. 2012) on board the Solar Dynamics Observatory (SDO; Pesnell et al. 2012), which is always discovering new phenomena, is largely contributing to a new era of fast (12 s), small-scale (pixel size of 0 .'6) observations of the solar corona. As a new step forward, the high-resolution coronal (Hi-C) instrument has provided hightemporal (5.5 s) and high-spatial (pixel size of 0.1 ) resolution observations of the EUV corona. Using this data set, Cirtain et al. (2013) have recently shown the existence of small-scale braided structures within the active region, and evidenced the magnetic reconnection process occurring within the braided structures. In addition, two recent articles relying on $\mathrm{Hi}-\mathrm{C}$ data have provided new insights in the existence of small-scale heating events. Testa et al. (2013) have found that the active region moss is highly dynamic with a variability on timescales of $15 \mathrm{~s}$, and the moss brightenings lead to an energy release of $10^{23}$ erg (order of magnitude consistent with the nanoflare model). Similarly, Winebarger et al. (2013) have investigated transient brightenings along small-scale transition-region loops. It was found that the loops have a diameter ranging from 0.9 to 1 .' 1 with an average duration of $64 \mathrm{~s}$. Winebarger et al. (2013) have estimated that these brightenings radiate an energy of about $10^{24}-10^{25} \mathrm{erg}$, which is another energy estimate consistent with the nanoflare model.

In this paper, we report on the existence of an additional heat source for the coronal plasma: EUV, short-lived (25 s), smallscale $\left(<1^{\prime \prime}\right)$ brightenings taking place in the low layers of the solar atmosphere $(\log T=5.5-6.5)$. We call these brightenings EUV bright dots (EBDs). This new discovery was made possible by the high spatial resolution and fast time cadence of the Hi-C data.

In Section 2, after introducing the Hi-C instrument, we characterize the location, size, and thermal properties of EBDs as derived from the Hi-C channel and six SDO/AIA channels. We thus study their time evolution in the observed region (Section 3), as well as their magnetic properties (Section 4), relying on a potential field extrapolation of one $S D O / \mathrm{HMI}$ magnetogram. The conclusions are drawn in Section 5.

\section{STRUCTURE OF EUV BRIGHT DOTS}

\subsection{The Hi-C Instrument}

The Hi-C imager was launched on a sounding rocket on 2012 July 11 (Kobayashi et al. 2013). The Hi-C passband isolates a very narrow window in EUV centered at $193 \AA$, taking images with a pixel size of $0^{\prime \prime} 1$. The intensity line

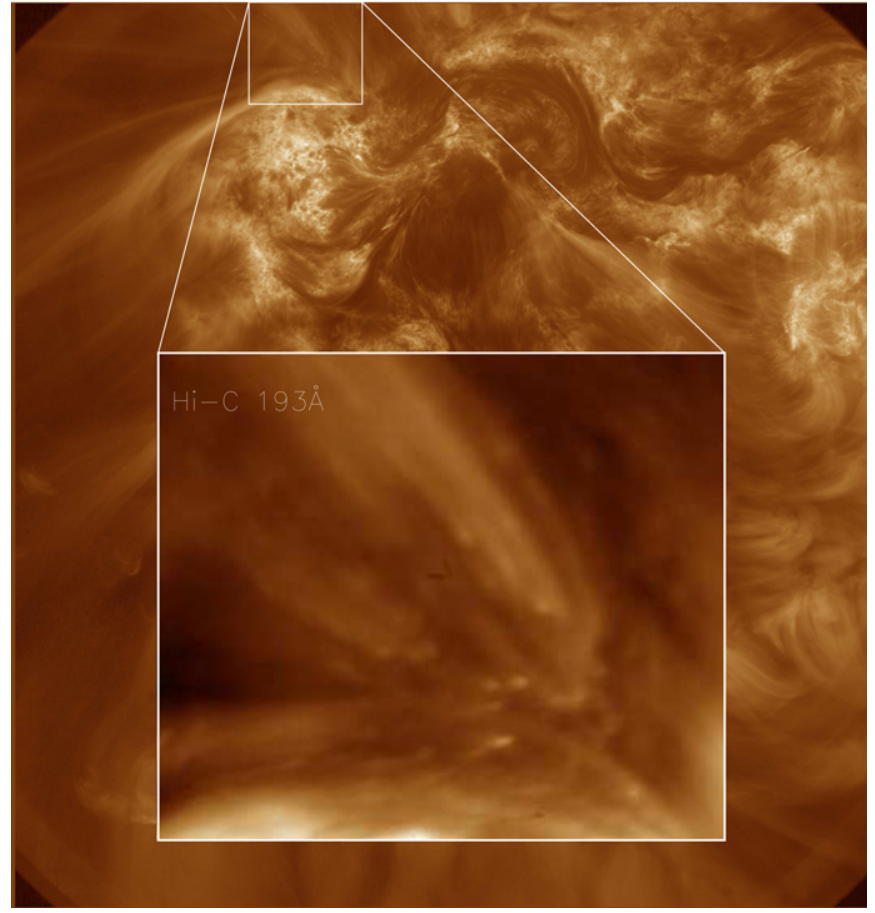

Figure 1. Location of the region of interest containing the EBDs in the Hi-C FOV recorded at 18:53:32 UT on 2012 July 11.

(A color version of this figure is available in the online journal.)

profile is dominated by an emission line of Fe XII with a peak temperature at $1.5 \mathrm{MK}(\log T=6.17)$, and blended by some weak transition region lines, as well as including 10-15 MK emission during large flares. The main target of the Hi-C instrument was the complex system of active regions located around the central meridian in the Southern hemisphere (active regions NOAA 11519-21), which mostly contains the active region 11520 (AR 11520). In Figure 1, we plot the full Hi-C field-of-view (FOV) centered approximately at $(-150,-281)$ arcsec from the Sun center. We extract the region of interest containing the EBDs discussed in this paper. The size of the EBDs' FOV is approximately $35^{\prime \prime} \times 30^{\prime \prime}$. Magnetically, AR 11520 is a collection of seven to eight sunspots as shown in Figure 10, left panel. The Hi-C time series lasts for $200 \mathrm{~s}$, from 18:52:09 UT to 18:55:29 UT, with a time cadence of $5.5 \mathrm{~s}$ (36 images).

\subsection{Comparing Hi-C and SDO/AIA Observations}

In Figure 2, we compare the EUV emission at $193 \AA$ observed in Hi-C (left) and SDO/AIA (right; Lemen et al. 2012). Even if they are centered on the same wavelength, the Hi-C and SDO/AIA filters are similar but not identical. The high spatial resolution of the $\mathrm{Hi}-\mathrm{C}$ observations provides further insights into the small-scale coronal structures and phenomena, which are observed as localized brightenings; EBDs that can be resolved in the Hi-C FOV are seen as compact bright regions in the SDO/ AIA FOV. To extract the signature of the EBDs, we proceed in three consecutive steps: (1) applying a median filter to a single frame, (2) considering the intensities above the $3 \sigma$ level (standard deviation of the intensity map in a single frame), and (3) adding the intensities of the frames together over the whole $\mathrm{Hi}-\mathrm{C}$ time series. We thus can define the locations of significant bright dots (see Figure 2, bottom-left panel), and subsequently study their time evolution and geometrical properties. Here, 

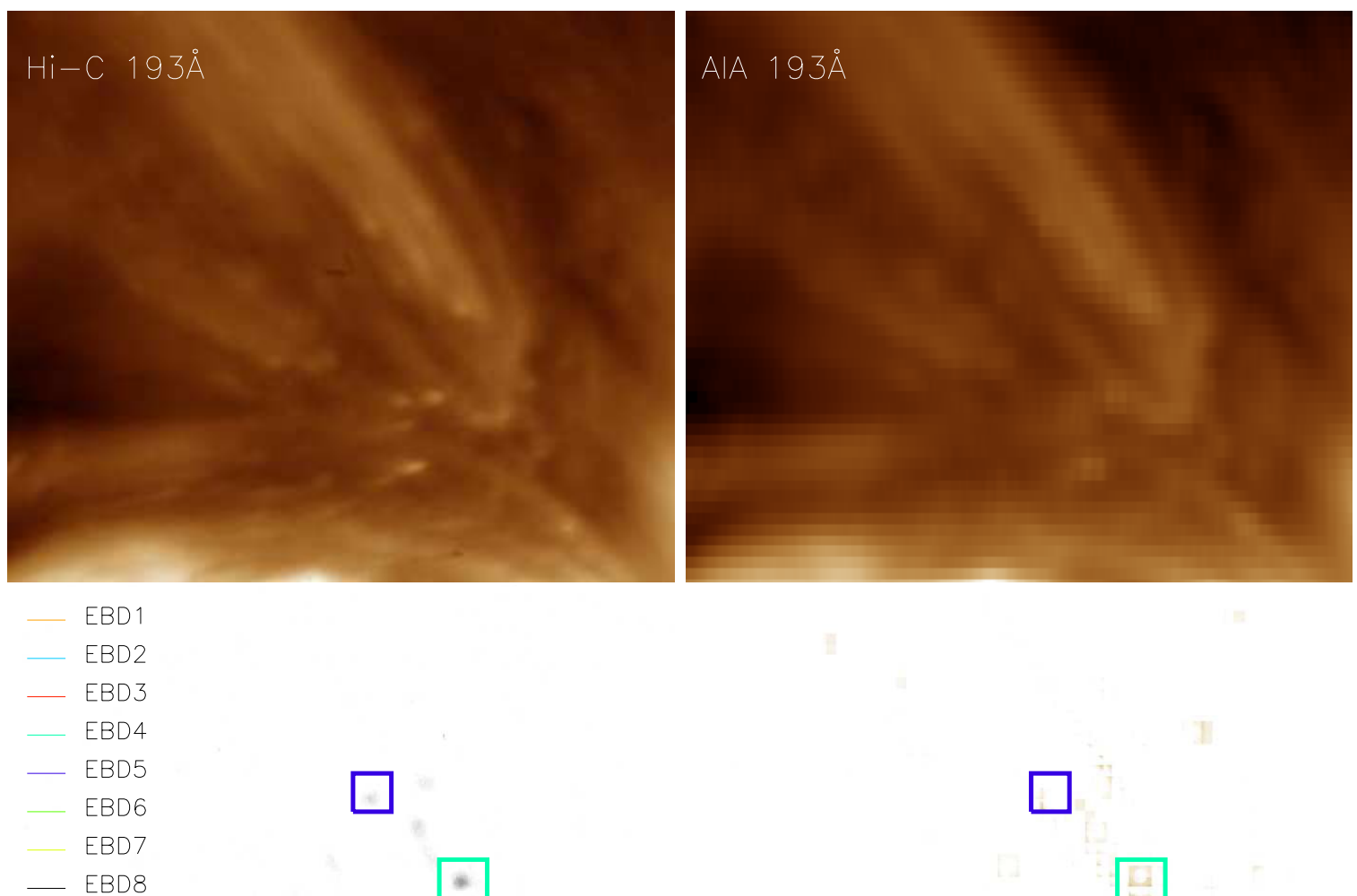

Figure 2. Top row: integrated intensities over the whole Hi-C time series observed in Hi-C at $193 \AA$ (left), and in SDO/AIA at $193 \AA$ (right). Bottom row: filtered and integrated intensities during the whole Hi-C time series: (left) Hi-C EBDs, (right) SDO/AIA EBDs.

(A color version of this figure is available in the online journal.)

eight significant bright dots are identified (as depicted by the rectangles in Figure 2, bottom-left panel). The eight different EBDs have been chosen because they are associated with a well-defined structure in individual images: elliptical structures of more than 9 pixels and with an intensity above the $3 \sigma$ level (standard deviation of a single frame). Even if they do not obey the criteria imposed above, other brightenings (not classified as EBDs) exist in the FOV, which suggest that smaller spatial-scale structures can exist.

In Figure 2 bottom right, the location of the EBDs selected in the Hi-C image (left) is compared to the SDO/AIA image (right) processed with the same methodology as the Hi-C data-most of the chosen EBDs can be identified in both FOVs. Solely based on the images, the signal is much weaker for the $S D O / A I A$ observations, and thus using $S D O /$ AIA observations alone would not provide confidence of their existence; only a comparison with the Hi-C images ensures that the SDO/AIA EBDs are true brightenings and not just noise.

\subsection{Multi-wavelength Observations of EBDs}

Using the capabilities of the $S D O /$ AIA instrument, we study the EBDs using six other wavelength channels covering the low chromosphere to the high temperature corona (1600 $\AA, 304 \AA$, $131 \AA$, $171 \AA, 211 \AA$, and $335 \AA$ ). In Figure 3, top panel, we plot the integrated images over the whole Hi-C time series in the different channels for the same FOV. In Figure 3, bottom panel, we apply the same data analysis procedure to the time series for the six wavelength channels in order to enhance the location of EBDs.

From Figure 3, we find the following possible signatures of EBDs:

1. the $1600 \AA$ channel does not show much evidence of the EBD intensity signature,

2. the $304 \AA$ channel shows weak emission for some of the EBDs, and

3. the hotter channels ( $131 \AA, 171 \AA, 211 \AA$, and $335 \AA$ ) show clear evidence of the EBD intensity signatures, as in the $193 \AA$ A channel.

Comparing the temperature response functions of $S D O /$ AIA and their overlapping areas (Boerner et al. 2012), this multichannel analysis suggests that the EBDs are in a temperature range between 0.3 and $1.5 \mathrm{MK}(\log (T)=5.5-6.2)$, which implies that the EBDs are either located at the top of the chromosphere or at the bottom of the corona.

\subsection{EBDs' Temperature: EM Loci}

In order to investigate the possible temperature of the EBDs, the EM loci technique was employed. We used the same 

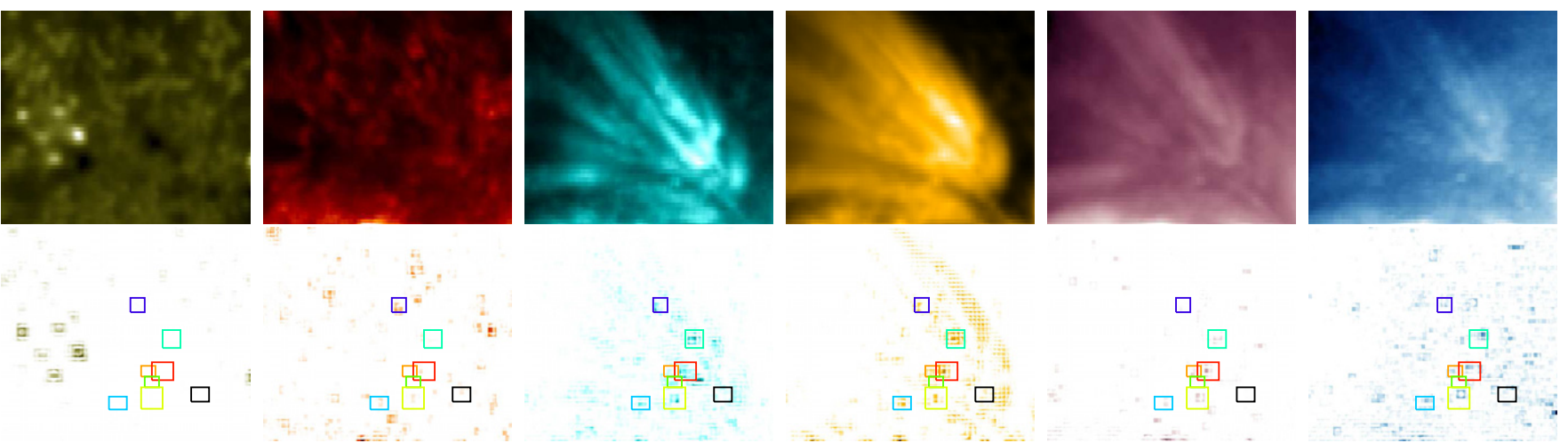

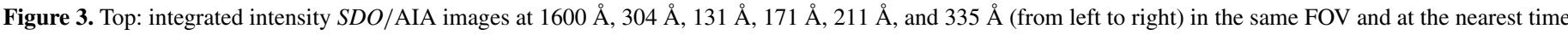
to the Hi-C image in Figure 2. Bottom: filtered images as in Figure 2, bottom panel (some color coding as in Figure 2).

(A color version of this figure is available in the online journal.)
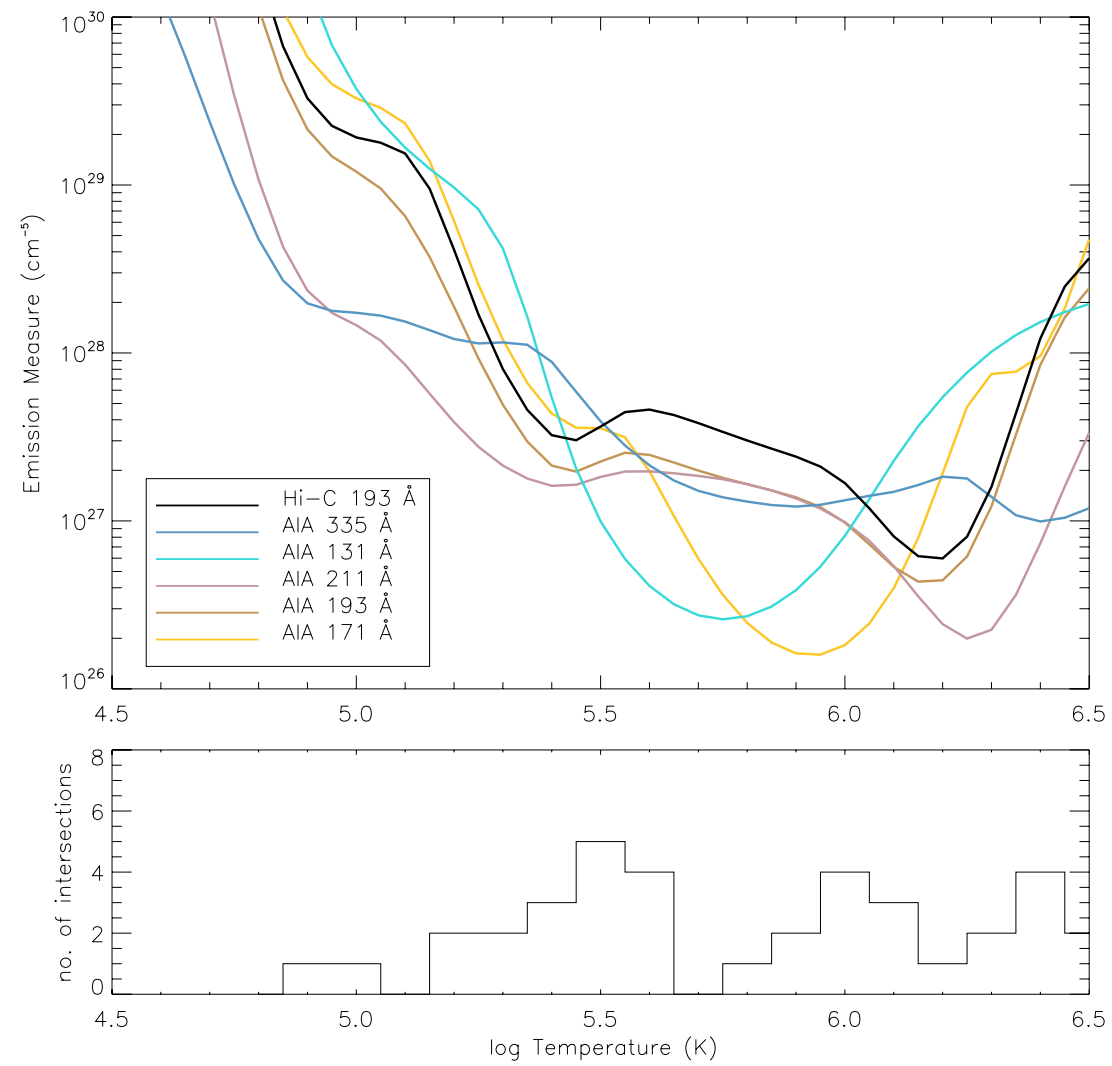

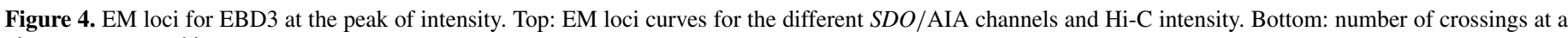
given temperature bin.

(A color version of this figure is available in the online journal.)

method as in Alexander et al. (2013), especially to get the temperature response functions of the different $S D O /$ AIA and Hi-C channels. The EM loci method (e.g., Jordan et al. 1987; del Zanna et al. 2002) allows us to determine if a coronal structure is isothermal or multi-thermal without providing more in-depth information (see Figure 7 in Reale 2010 for an example of isothermal and multi-thermal loops). We derived the EM loci curves of several SDO/AIA channels (131 A, $171 \AA$, $193 \AA$, $211 \AA$, and $335 \AA$ ) and the $193 \AA$ Hi-C channel. A single crossing of all the EM loci curves should indicate an isothermal plasma at this temperature.

In Figure 4, we present the results of the EM loci method for EBD3. There is clear evidence that three possible temperature regions, which correspond to the larger number of loci crossings, may be distinguished - one at low temperature, with $\log (T)=$ 5.5 , and two at coronal temperature, with $\log (T)=6.0$ and $\log (T)=6.4$. However, for all other EBDs studied, the EM loci method does not lead to any conclusive temperature, with the crossings being distributed between $\log (T)=5.3$ and 6.5. The lack of clarity in the EM loci curves may be the result of two points, either (1) the size and timing of the EBDs' structure at the $S D O /$ AIA scale do not permit any firm conclusions to be drawn (as noted in Section 2, the EBDs are very difficult to observe in $S D O /$ AIA data), or (2) the plasma of the EBDs is a transition region plasma for which a temperature cannot be determined by the lines observed. The latter implies that the mechanism to 
Table 1

Characteristic Parameters of EBDs

\begin{tabular}{|c|c|c|c|c|c|c|}
\hline \multirow[t]{2}{*}{ Cat. } & \multirow[t]{2}{*}{ Name } & \multirow{2}{*}{$\begin{array}{l}\text { Peak time } \\
t_{\text {peak }}(\mathrm{UT})\end{array}$} & \multirow[t]{2}{*}{$\Delta I$} & \multicolumn{2}{|c|}{ Duration (s) } & \multirow{2}{*}{$\begin{array}{l}\text { Length } \\
(\mathrm{Mm})\end{array}$} \\
\hline & & & & $\Delta_{\text {peak }}$ & $\Delta_{v}$ & \\
\hline \multicolumn{7}{|c|}{ Single } \\
\hline \multirow[t]{3}{*}{ I } & EBD1 & $18: 52: 48$ & 1.68 & 27 & 64 & 0.65 \\
\hline & EBD2 & $18: 52: 59$ & 2.00 & 39 & 61 & 0.5 \\
\hline & EBD3 & $18: 54: 06$ & 3.89 & 22 & 89 & 0.86 \\
\hline \multicolumn{2}{|c|}{ Average values } & & 2.52 & $29 \mathrm{~s}$ & $71 \mathrm{~s}$ & $0.67 \mathrm{Mm}$ \\
\hline \multicolumn{7}{|c|}{ Double-peak } \\
\hline \multirow[t]{6}{*}{ II } & EBD4 & $18: 52: 59$ & 1.99 & 28 & 61 & 0.43 \\
\hline & & $18: 53: 16$ & 1.82 & 22 & & 0.76 \\
\hline & EBD5 & $18: 53: 10$ & 1.55 & 23 & 56 & 0.76 \\
\hline & & $18: 53: 43$ & 1.52 & 28 & & 0.72 \\
\hline & EBD6 & $18: 54: 56$ & 1.76 & 16 & 83 & 0.65 \\
\hline & & $18: 55: 12$ & 1.84 & 22 & & 0.76 \\
\hline \multicolumn{2}{|c|}{ Average values } & & 1.75 & $23 \mathrm{~s}$ & $67 \mathrm{~s}$ & $0.68 \mathrm{Mm}$ \\
\hline \multicolumn{7}{|c|}{ Long Duration } \\
\hline \multirow[t]{6}{*}{ III } & EBD7 & $18: 52: 31$ & 1.34 & 23 & 200 & $\mathrm{D}$ \\
\hline & & $18: 52: 59$ & 1.70 & 23 & & 0.43 \\
\hline & & $18: 53: 43$ & 1.98 & 28 & & 0.79 \\
\hline & & $18: 54: 17$ & 2.06 & 23 & & 0.79 \\
\hline & & $18: 54: 45$ & 1.93 & 5 & & 0.57 \\
\hline & & $18: 55: 01$ & 2.38 & 16 & & 0.79 \\
\hline \multicolumn{2}{|c|}{ Average values } & & 1.89 & $20 \mathrm{~s}$ & $200 \mathrm{~s}$ & $0.67 \mathrm{Mm}$ \\
\hline \multicolumn{7}{|c|}{ Bursty } \\
\hline \multirow[t]{5}{*}{ IV } & EBD8 & $18: 53: 21$ & 1.28 & 5 & 133 & 0.43 \\
\hline & & $18: 53: 43$ & 1.32 & 5 & & 0.43 \\
\hline & & $18: 54: 00$ & 1.47 & 5 & & 0.43 \\
\hline & & $18: 54: 45$ & 1.44 & 5 & & 0.43 \\
\hline & & $18: 54: 56$ & 1.44 & 5 & & 0.43 \\
\hline \multicolumn{2}{|c|}{ Average values } & & 1.39 & $5 \mathrm{~s}$ & $133 \mathrm{~s}$ & $0.43 \mathrm{Mm}$ \\
\hline
\end{tabular}

release magnetic energy most likely occurs at the footpoints of the coronal loops. The behavior of the EM loci method for the EBDs as observed by $S D O /$ AIA is consistent with the study of the multi-thermal plasma performed by Guennou et al. (2012).

\section{CHARACTERISTICS AND DYNAMICS OF EBDS}

\subsection{Categories of EBDs}

We analyze the Hi-C light curves and images of the eight selected EBDs within the areas depicted in Figure 2, bottom-left panel. In Table 1, we give the main observed characteristics for the eight chosen EBDs, including the time of the peak intensity $t_{\text {peak }}$; the relative intensity $\Delta I$ of the maximum intensity at $t_{\text {peak }}$ with respect to the average intensity over the full EBD FOV at the same time; the duration $\Delta_{\text {peak }}$ from the light curve given by the width at half-maximum of the intensity peak (with respect to the minimum intensity of the light curve); and $\Delta_{v}$ the time during which a coherent structure (elliptical surface of intensity covering more than 9 pixels) can be identified within the time series, and the characteristic length (major axis of the ellipse) of the EBDs. The area considered for individual EBDs is the size of the rectangle depicted in Figure 2 bottom left.

From Table 1, we deduce that, on average, EBDs have a characteristic lifetime of $25 \mathrm{~s}$, with a characteristic length of $0.68 \mathrm{Mm}$.

From the light curves of the identified EBDs, we distinguish four categories of EBDs based on their frequency within the Hi-C time series (see Figures 5-8, top panel, and also Table 1):
I. Single peak: single events with a clear peak in the light curve. EBD1-3 have durations between 22 and 39 s, and characteristic sizes between 0.5 and $0.86 \mathrm{Mm}$;

II. Double peak: double-peaked EBDs with two consecutive maxima. EBD4-6 have two peaks separated by 17-33 s, with a short global duration (about $67 \mathrm{~s}$ );

III. Long duration: long-duration EBDs for which the coherent structure can be characterized during the entire time series (200 s), and with several distinct maxima. For EBD7, six intensity peaks are observed within the Hi-C time series, with a time interval from $16 \mathrm{~s}$ to $44 \mathrm{~s}$. In Table 1, the value "D" means that the EBD intensity distribution is diffuse;

IV. Bursty: a series of very short brightenings related to a coherent structure, and leading to an increase of the overall intensity. The EBD8 light curve shows an increase of intensity starting at 18:53:16 UT, and has several short peaks ( $5 \mathrm{~s}$ ) observed with a cadence from $11 \mathrm{~s}$ to $45 \mathrm{~s}$.

From this small sample of events, Categories I and II are the most significant. We now proceed to a detailed analysis of one single EBD in each of the four categories. For Categories I and II, the EBD analyzed has the largest intensity contrast ( $\Delta I$ in Table 1).

\subsection{Category I: Single Peak}

Consider EBD3; in Figure 5, top panel, we plot the HiC normalized light curve of EBD3, which exhibits a clearly distinctive peak in intensity. The peak lasts for five frames (22 s). We also plot the normalized light curves for different $S D O /$ AIA 

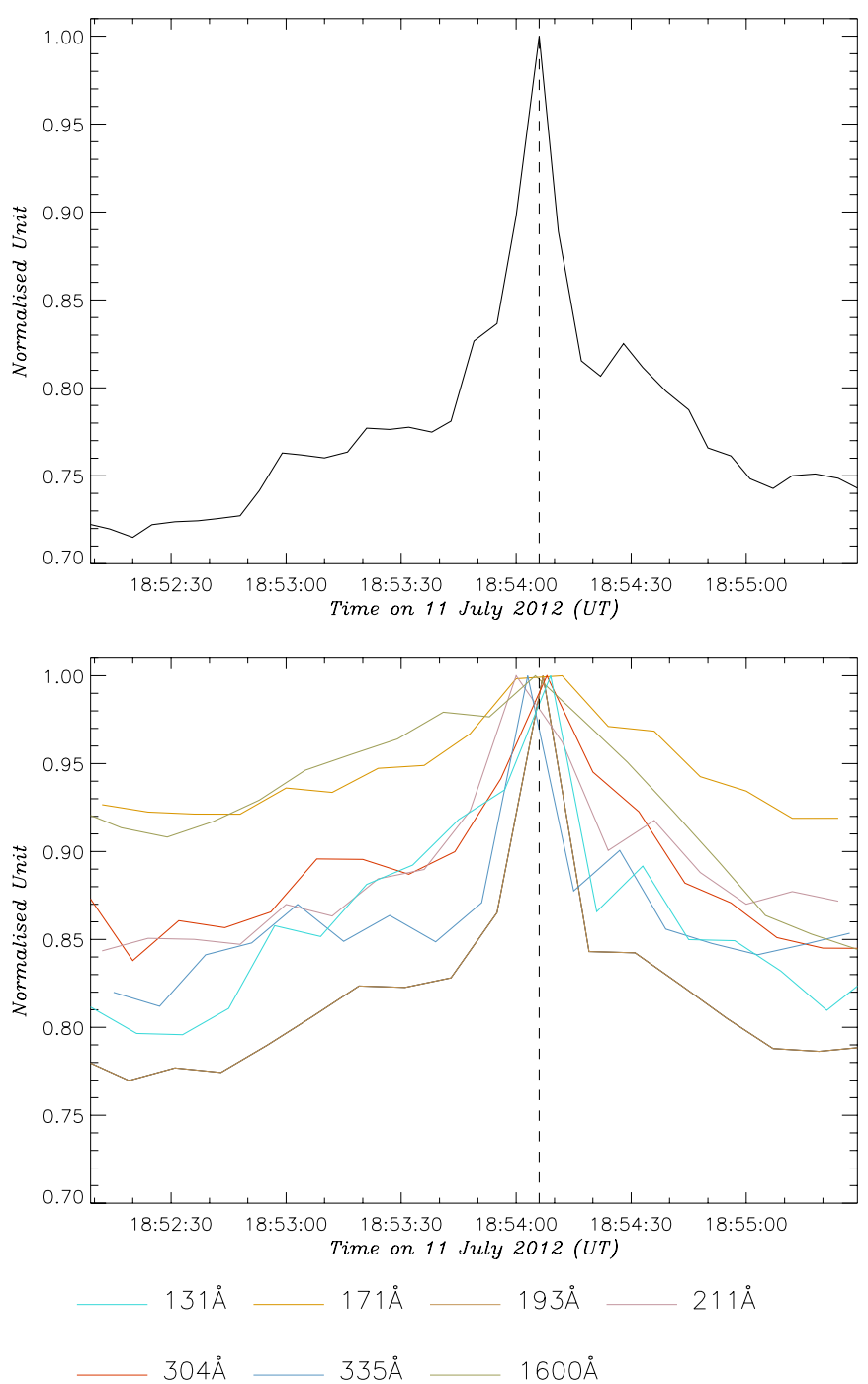

Figure 5. Top: the Hi-C normalized light curve for EBD3 clearly showing the single peak of intensity characterizing the bright dot. Bottom: SDO/AIA normalized light curves for the same area encompassing EBD3 in seven different EUV-UV channels ( $131 \AA$ A, $171 \AA$ A, $193 \AA, 211 \AA, 304 \AA, 335 \AA$, and $1600 \AA$ ).

(A color version of this figure is available in the online journal.)

channels (see Figure 5, bottom panel), where we distinguish a corresponding peak in intensity (see Figure 9(a)). Due to the short duration of the EBDs $(\sim 22 \mathrm{~s})$ and the time cadence of $S D O /$ AIA images $(12 \mathrm{~s})$, it is difficult to conclude when the peak emission has actually occurred in the different channel. As shown by Viall \& Klimchuk $(2011,2012)$, the cooling of a nanoflare storm within a coronal loop may be deduced by the order of the appearance of intensity peaks in the SDO/AIA light curves. This result follows the observational work done by Winebarger et al. (2003) using TRACE data. Such analysis cannot be performed here.

\subsection{Category II: Double Peak}

In Figure 6, top panel, the normalized Hi-C light curve for EBD4 clearly shows two distinct consecutive peaks (see Figure 9(b)): each individual peak has the property of the EBDs of Category I. In Figure 6, bottom panel, the peaks are also observed in the $193 \AA$ and $171 \AA$ SDO/AIA channels. The first peak at 18:52:59 UT is clearly observed in the other channels, while the second peak cannot be identified.
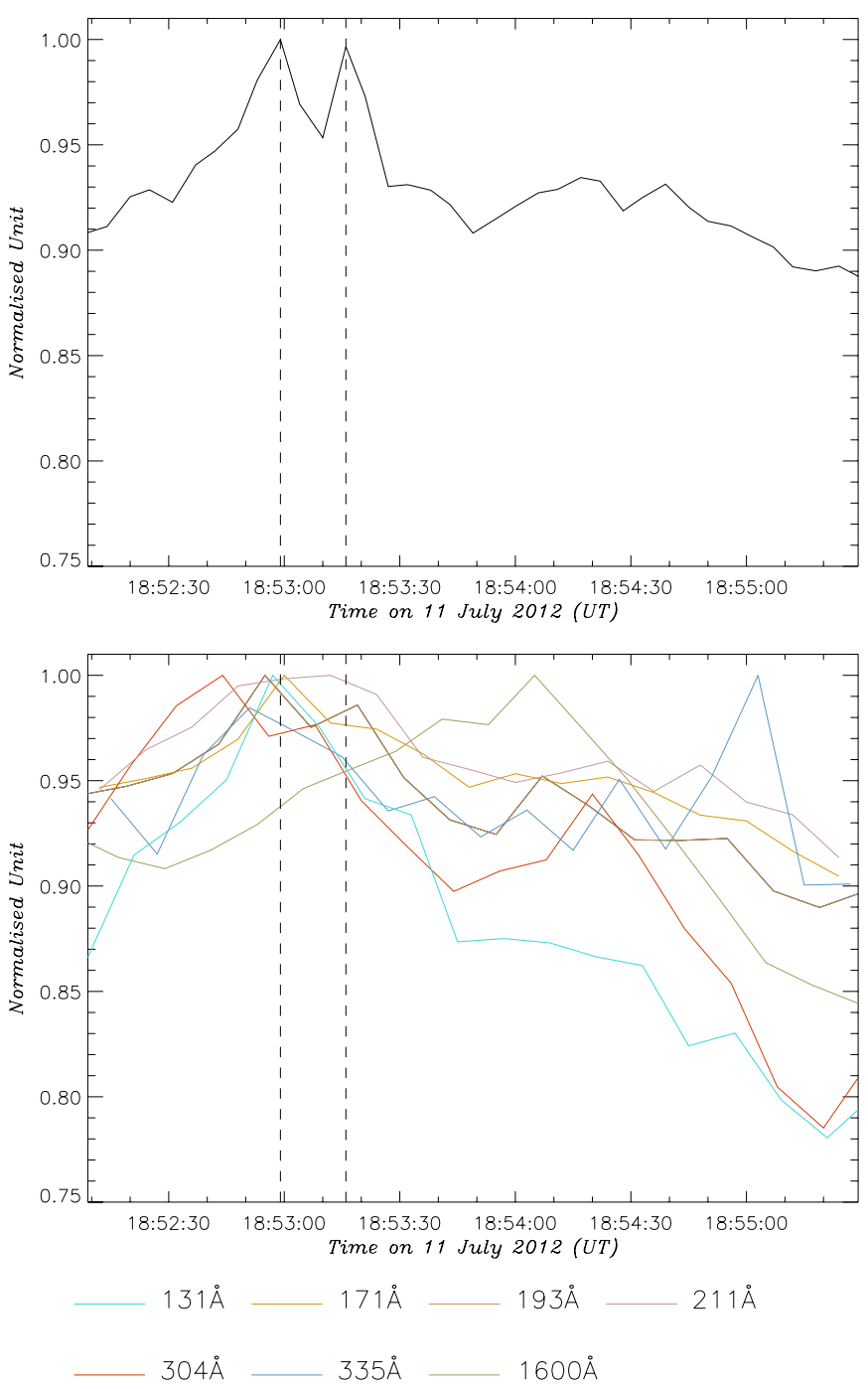

Figure 6. Same as Figure 5 for EBD4 in Category II.

(A color version of this figure is available in the online journal.)

\subsection{Category III: Long Duration}

In Figure 7, we plot the Hi-C-normalized light curve (top) and the $S D O$ /AIA light curves (bottom) for EBD7, the only example of Category III EBDs. The Hi-C light curve shows several intensity peaks that can be observed during the whole time period. The peaks (marked by vertical dashed lines) appear at 18:52:31 UT, 18:52:59 UT, 18:53:43 UT, 18:54:17 UT, 18:54:45 UT, and 18:55:01 UT. These Hi-C peaks are also observed in the $193 \AA$ SDO/AIA channel, which has a comparable mean intensity value, and most of them can be seen in $131 \AA$ and $171 \AA$. We also notice that the normalized light curves of $211 \AA$, $304 \AA$, and $335 \AA$ exhibit a similar bursty behavior, but at different times. EBD7 is observed as a coherent structure during the whole Hi-C time series (see Figure 9(c)). It is also possible to observe a slight displacement of the center of mass of EBD7, which leads to an apparent transverse velocity of $4.2 \mathrm{~km} \cdot \mathrm{s}^{-1}$ for a duration of $106 \mathrm{~s}$. The EBDs recur at a period of about $30 \mathrm{~s}$.

\subsection{Category IV: Bursty}

In Figure 8, we see a clear increase in intensity from 18:53:16 UT to the end of the Hi-C time series, as several peaks are observed during this period. The identified peaks appear at 

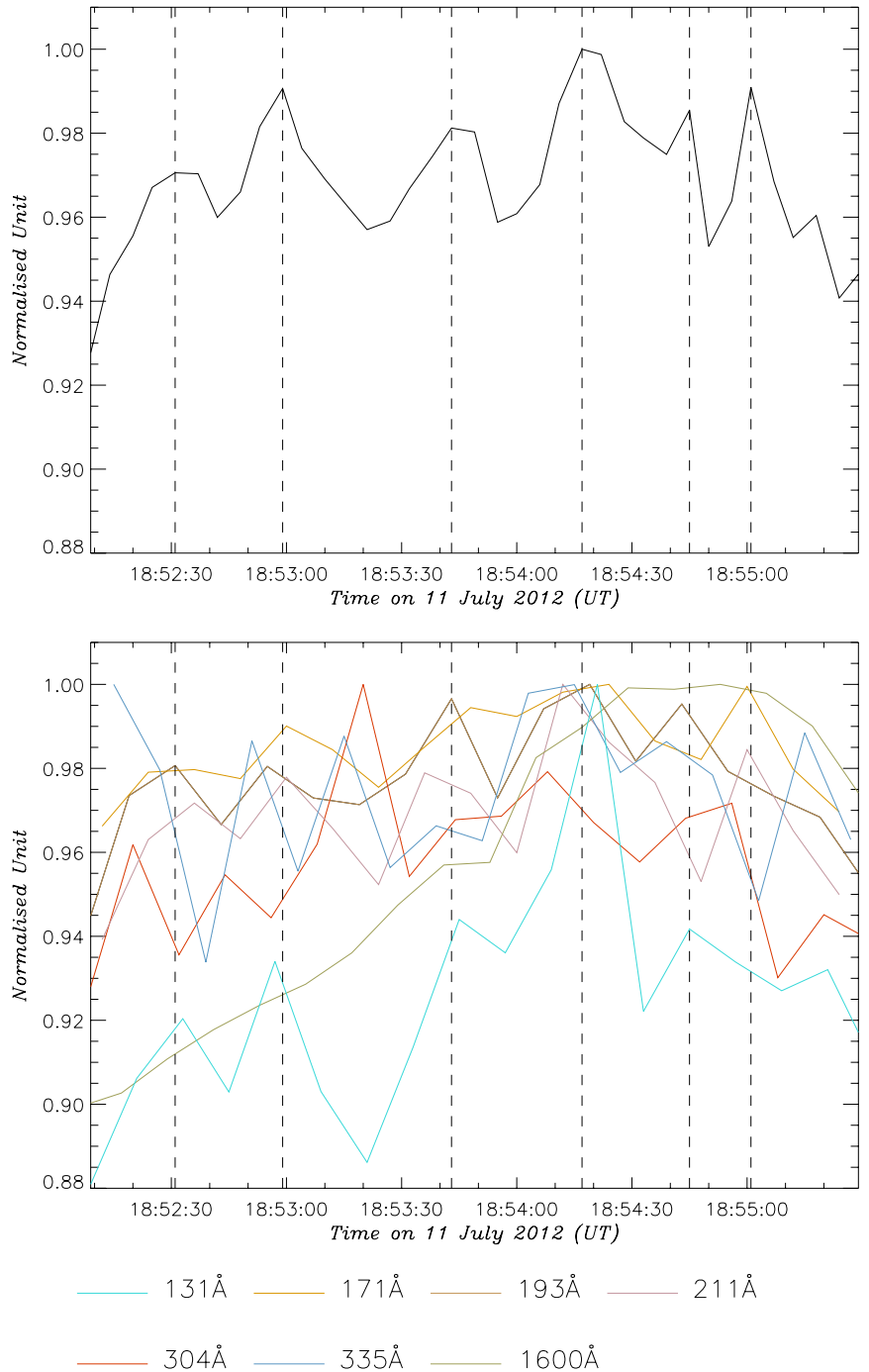

Figure 7. Same as Figure 5 for EBD7 in Category III.

(A color version of this figure is available in the online journal.)

18:53:21 UT, 18:53:43 UT, 18:54:00 UT, 18:54:45 UT, and 18:54:56 UT (see also Figure 9(d)). The peaks are not all seen in the $193 \AA S D O /$ AIA channel due to their short duration and the timespan between consecutive SDO/AIA images. The EBDs recur at a period of about $20 \mathrm{~s}$. This short period and the increase of intensity suggest that the energy released during the EBDs heats up the plasma without the possibility for the conductive cooling to take place.

\section{MAGNETIC FIELD STRUCTURE}

\subsection{SDO/HMI Data and Extrapolated Magnetic Field Over the Hi-C FOV}

A line-of-sight magnetogram provided by $S D O / \mathrm{HMI}$ (Scherrer et al. 2012) allows us to extrapolate the potential magnetic field into the solar atmosphere. The SDO/HMI magnetogram was captured on 2012 July 11 at 18:54:00 UT, with a pixel size of 0.5 .

The potential field assumption considers that

$$
\boldsymbol{\nabla} \times \boldsymbol{B}=\mathbf{0} .
$$

The system of differential equations is solved as a boundary value problem in defining the normal component of the magnetic
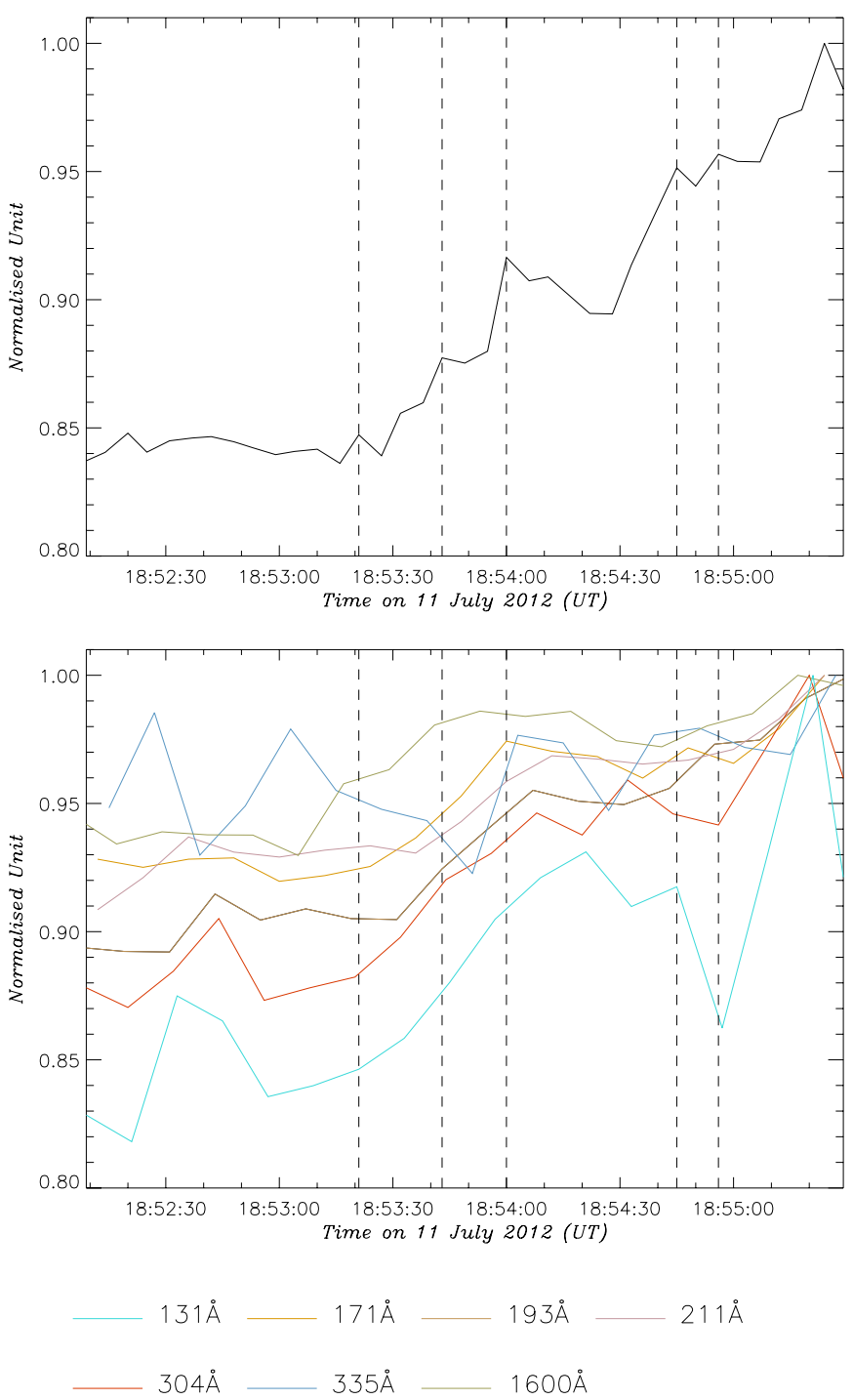

Figure 8. Same as Figure 5 for EBD8 in Category IV.

(A color version of this figure is available in the online journal.)

field on each surface of the computational box. Two types of potential fields are computed: (1) the potential field for which the original SDO/HMI magnetogram (see Figure 10, left panel) is used as the bottom boundary condition, and open boundary conditions on the sides and top of the computational box are applied; or (2) the fully open potential field within the computational box (as defined by Aly 1984) for which the magnetic field at the bottom boundary is unipolar. The first extrapolated magnetic field corresponds to a minimum of magnetic energy for this set of normal magnetic field distributions, whereas the second model corresponds to an upper bound of magnetic energy for a force-free field (Aly 1984). Thus the difference between the magnetic energies derived from both models gives an upper bound for the free magnetic energy contained in the magnetic configuration.

In Figure 10, left panel, the selected SDO/HMI FOV can be seen $\left(144 \times 126 \mathrm{Mm}^{2}\right)$, which includes most of AR 11520 and for which the total unsigned magnetic flux is nearly balanced (see Table 2). In Figure 10, right panel, we plot the Hi-C FOV corresponding to the location of the EBDs. Subsequently, in Table 2, we summarize the magnetic properties of the full 

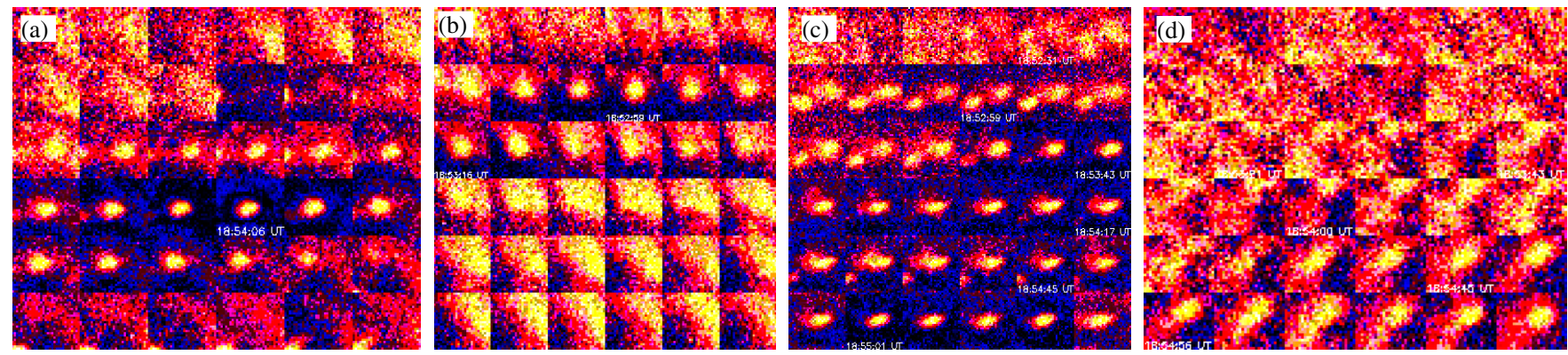

Figure 9. Time evolution of the intensity associated with the four EBDs studied: (a) EBD3 (FOV: 3". $1 \times 2$ 2"6), (b) EBD4 (FOV: 2". $6 \times 2$ ".6), (c) EBD7 (FOV: 3". $1 \times$ 3'.1), and (d) EBD8 (FOV: 2". $6 \times 2$ 2' 1). Time increases from left to right, and top to bottom. The time of the peaks marked on the light curves is annotated on the images. The images have been normalized to the maximum EBD intensity in the Hi-C time series.

(A color version of this figure is available in the online journal.)
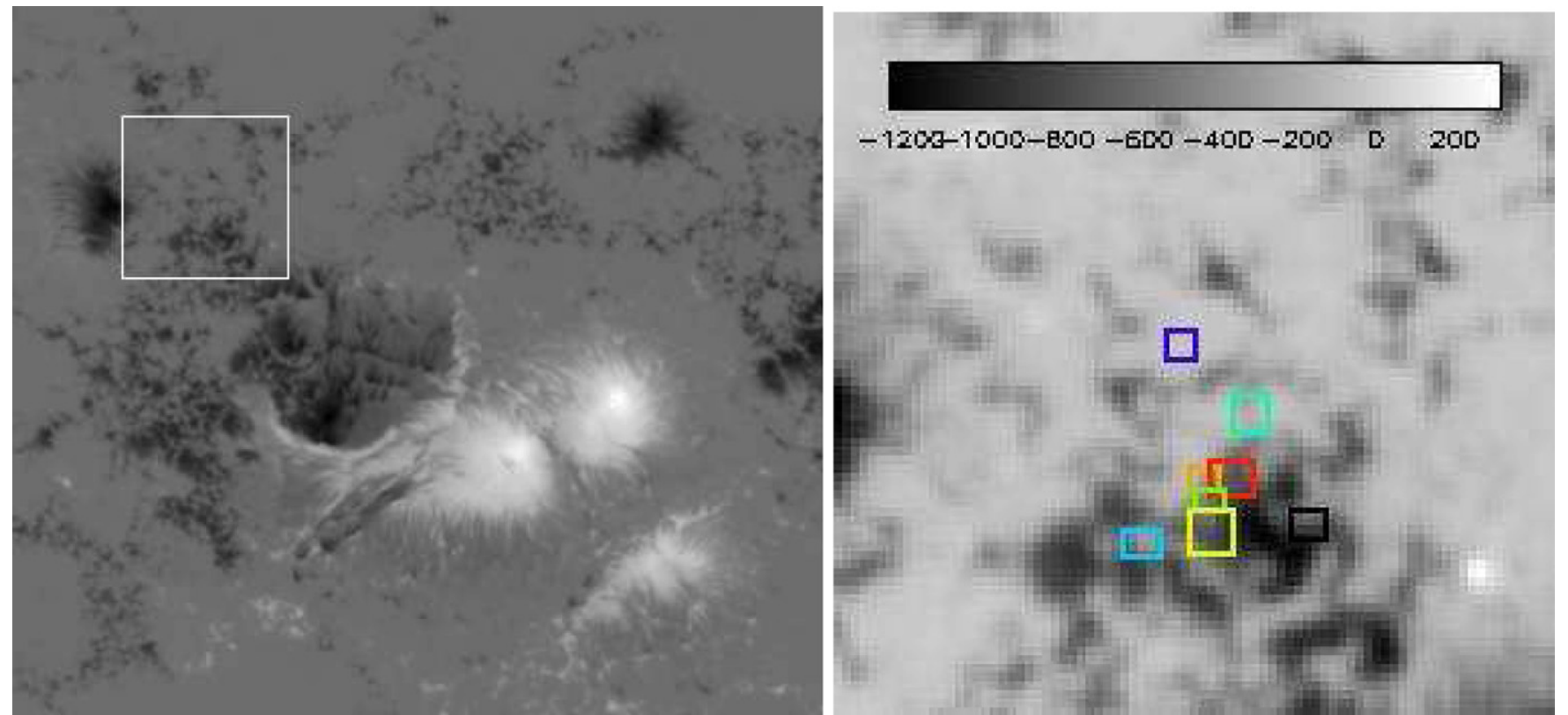

Figure 10. Left: line-of-sight magnetic field distribution in the full Hi-C FOV observed by SDO/HMI. The EBD FOV is indicated by the white rectangle. Right: close-up of the magnetic field in the area where the EBDs are observed (same color coding as in Figure 2, bottom panel). The line-of-sight magnetic field strength (G) is indicated by the color bar.

(A color version of this figure is available in the online journal.)

Table 2

Magnetic Properties

\begin{tabular}{lcccccccc}
\hline \hline & $\begin{array}{c}B_{\text {los }}^{\min } \\
(\mathrm{G})\end{array}$ & $\begin{array}{c}B_{\text {los }}^{\max } \\
(\mathrm{G})\end{array}$ & $\begin{array}{c}\phi_{T} \\
(\mathrm{Mx})\end{array}$ & $\begin{array}{c}\phi_{\text {net }} \\
(\mathrm{Mx})\end{array}$ & $\begin{array}{c}\phi_{+} \\
(\mathrm{Mx})\end{array}$ & $\begin{array}{c}\phi_{-} \\
(\mathrm{Mx})\end{array}$ & $\begin{array}{c}E_{\mathrm{pot}} \\
(\mathrm{erg})\end{array}$ & $\begin{array}{c}E_{\text {open }} \\
(\mathrm{erg})\end{array}$ \\
\hline Full FOV & -1830 & 2463 & $5.97 \times 10^{22}$ & $-9.00 \times 10^{21}$ & $2.53 \times 10^{22}$ & $-3.43 \times 10^{22}$ & $1.68 \times 10^{33}$ & $3.03 \times 10^{33}$ \\
EBD FOV & -1232 & 316 & $2.61 \times 10^{21}$ & $-2.60 \times 10^{21}$ & $7.43 \times 10^{18}$ & $-2.60 \times 10^{21}$ & $5.20 \times 10^{31}$ & $8.98 \times 10^{31}$ \\
\hline
\end{tabular}

FOV used to perform the potential field extrapolation, and then reduced the EBD FOV:

1. the minimum and maximum line-of-sight magnetic field strength for the photospheric magnetogram $\left(B_{\mathrm{los}}^{\min }, B_{\mathrm{los}}^{\max }\right.$, respectively);

2. the total unsigned magnetic flux $\left(\phi_{T}\right)$,

$$
\phi_{T}=\int_{S}\left|B_{z}\right| d S
$$

where $S$ is the photospheric surface;

3 . the net magnetic flux $\left(\phi_{\text {net }}\right)$,

$$
\phi_{\text {net }}=\int_{S} B_{z} d S
$$

4. the positive and negative magnetic fluxes $\left(\phi_{+}, \phi_{-}\right.$, respectively):

$$
\phi_{+}=\int_{\Omega^{+}} B_{z} d S \quad \phi_{-}=\int_{\Omega^{-}} B_{z} d S
$$

where $\Omega^{+}$and $\Omega^{-}$are the domains of positive and negative $B_{z}$, respectively, on the photospheric surface;

5. the magnetic energy derived from the potential magnetic field with open boundary conditions $\left(E_{\mathrm{pot}}\right)$ and from the fully open magnetic field $\left(E_{\text {open }}\right)$. By definition, the magnetic energy $E_{m}$ for a magnetic field vector $B$ is given by

$$
E_{m}=\int_{V} \frac{B^{2}}{2 \mu_{0}} d V
$$




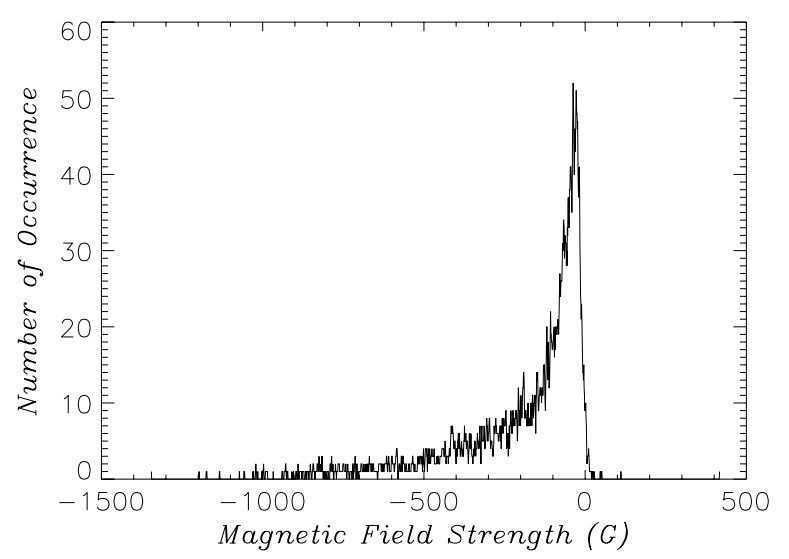

Figure 11. Histogram of the line-of-sight magnetic field strength in the region depicted in Figure 10, right panel.

where $V$ is the volume of the computational box and $\mu_{0}$ is the vacuum permeability.

Note that the full FOV has the total unsigned magnetic flux passing through the photosphere almost balanced, while the magnetic flux for the EBD FOV is dominated by the negative flux. As can be seen in Figure 11, the histogram of the lineof-sight magnetic-field strength in the EBD area shows that the negative polarity is dominant in this area and is (almost) unipolar. However, it is worth noting that the negative polarity where the EBDs are observed is not located in a sunspot (concentrated intense unipolar magnetic field), but instead in a plage magnetic field region (diffuse magnetic field that can contain parasitic polarities), so we cannot rule out the presence of small-scale positive polarities, which cannot be resolved by $S D O / \mathrm{HMI}$ and can play an important role in terms of low-lying magnetic reconnection events.

In Figure 12, we plot a selection of magnetic field lines that describe the global magnetic geometry of AR 11520 derived from a potential field approximation. Figure 12 highlights the magnetic connectivity between the sunspots of AR 11520 and outside (west side) toward AR 11519 and AR 11521. We also plot a significant number of magnetic field lines in and near the area containing the EBDs: this shows that those magnetic field lines are open (i.e., leaving the computational box) toward the east and north sides. It is worth noting that the magnetic field lines oriented toward the north are associated with transequatorial loops connecting to the positive polarity of a diffuse, decaying active region in the northern hemisphere.

Table 2 displays the magnetic energy of the potential and open magnetic field configuration for both the full FOV and EBD FOV. The magnetic energy of the full FOV is typical for an active region with a photospheric flux of the order of $10^{22}$ Mx. The upper bound for the free magnetic energy is estimated to be $E_{\text {open }}-E_{\text {pot }}=1.35 \times 10^{33} \mathrm{erg}$, which is consistent with the high-level of activity in AR 11520 for a couple days around the Hi-C observations (including an X1.4 flare on 2012 July 12). The upper bound for the free magnetic energy in the EBD FOV is estimated to $3.78 \times 10^{31} \mathrm{erg}$. Therefore, energy-release events such as a nanoflare (with an energy of $10^{24} \mathrm{erg}$ ) can be generated within the EBD FOV.

\subsection{EBD's Magnetic Field Structure}

We now study the magnetic properties of individual EBDs considering the same four events as in Section 3. For the sake of clarity, in Figure 13, we only plot a selection of magnetic field

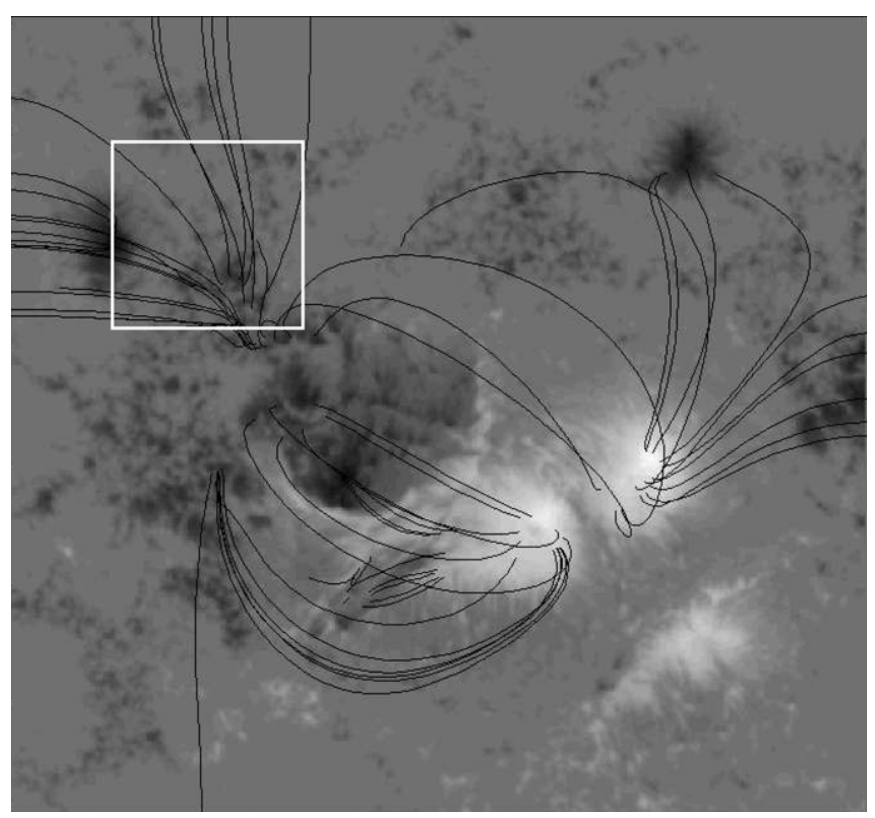

Figure 12. Selection of magnetic field lines within AR 11520 extracted from the potential field extrapolation. The background image is the line-of-sight magnetic field observed by $S D O / \mathrm{HMI}$. The white box indicates the FOV containing the EBDs as in Figure 10.

lines associated with EBD3 (Cat I), EBD4 (Cat II), EBD7 (Cat III), and EBD8 (Cat IV). For these EBDs, the magnetic field lines are leaving the computation box toward the northeast, consistent with the observed transequatorial loops connecting two active regions (see Section 4.1). We extract the magnetic information for each set of magnetic field lines: it is above $1 \mathrm{Mm}$ that the magnetic field strength becomes a smoothly decaying function of height. Below $1 \mathrm{Mm}$, the magnetic field strength is influenced by the complexity of the magnetic field and the change in orientation of the transverse component (parallel to the photosphere). It is also below $1 \mathrm{Mm}$ that the magnetic field lines plotted in Figure 13 can be considered as radial. The magnetic field strength of the four EBDs below $1 \mathrm{Mm}$ varies between $200 \mathrm{G}$ and $280 \mathrm{G}$.

We estimate the magnetic energy associated with the EBDs by considering the potential field within a sphere of diameter $0.68 \mathrm{Mm}$ (the characteristic size of EBDs) and within $1 \mathrm{Mm}$ above the photosphere, where the magnetic field strength has been estimated between 200 and $280 \mathrm{G}$. The magnetic energy is thus between $1.98 \times 10^{26}$ and $3.87 \times 10^{26} \mathrm{erg}$. An upper bound for the free magnetic energy is about $80 \%$ of the previous values (i.e., $1.58-3.1 \times 10^{26} \mathrm{erg}$ ).

The free magnetic energy estimates are of $10^{26} \mathrm{erg}$, which are similar to the energy associated with a single micro-flare. Thus, the magnetic field associated with the EBDs contains enough free magnetic energy to power single or multiple nanoflares as envisioned by Parker (1988), knowing that a small part of the free magnetic energy is commonly released during flares (e.g., Amari et al. 2000). By invoking an energy release site reduced by a factor of $3(0.23 \mathrm{Mm}$ instead of $0.68 \mathrm{Mm})$, the magnetic energy estimated reaches the nanoflare scale. This magnetic energy estimate evidences that the nanoflare model can be a viable mechanism for releasing magnetic energy at low heights in the solar atmosphere and thus heat the coronal part of the loop. 

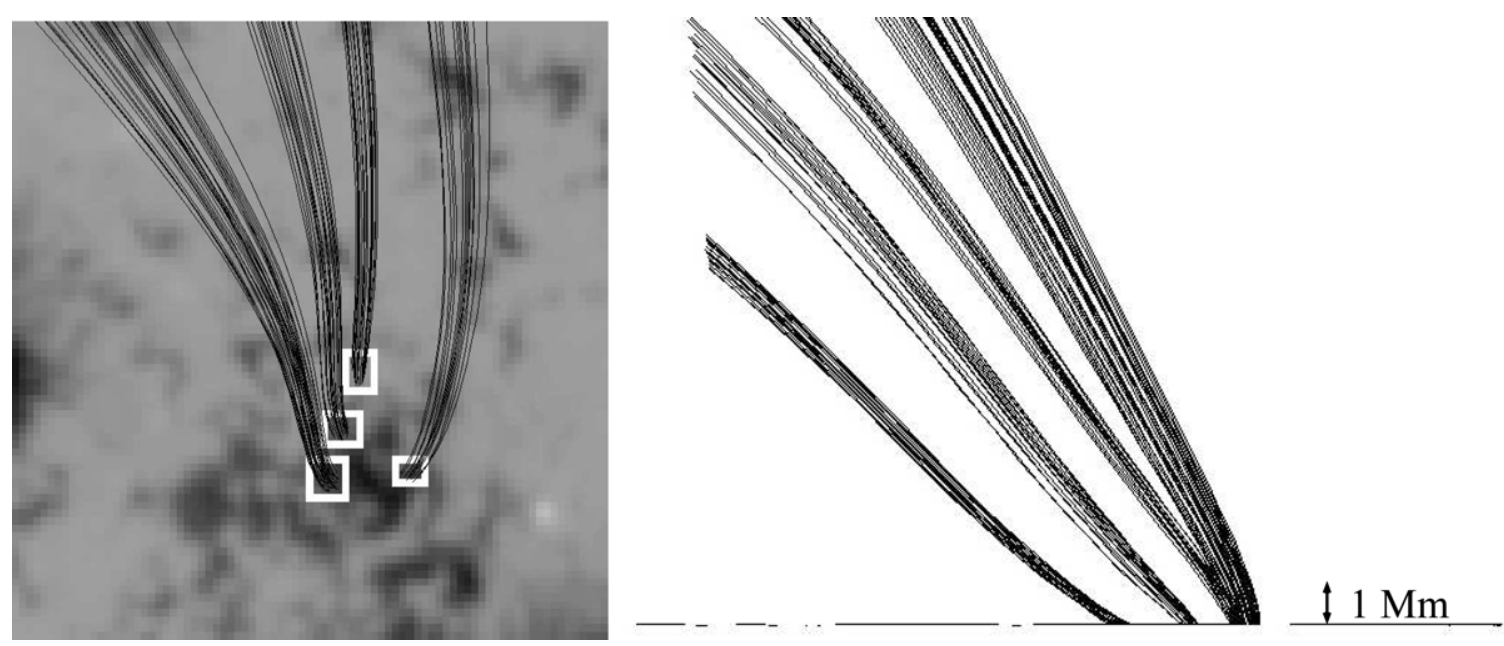

Figure 13. Selection of magnetic field lines for the four EBDs studied in Section 4.2. Left: top view with the EBDs' location depicted by white rectangles (see Figure 2). Right: view from the east side (north is on the left-hand side)

\section{CONCLUSIONS}

The high resolution images obtained in the EUV by $\mathrm{Hi}-\mathrm{C}$ allow us to determine the smallest brightenings observed to date in an active region in extreme ultraviolet light, called EBDs. These EBDs sparkle at the footpoints of large-scale coronal loops. We have grouped the EBDs into four categories depending on their recurrence. Except for the fourth category (successive, short-lived EBDs), individual EBDs that can be extracted from the Hi-C time series have the same characteristic duration (about $25 \mathrm{~s}$ ) and length scale $(0.68 \mathrm{Mm})$. Using a potential field extrapolation of the $S D O / \mathrm{HMI}$ line-of-sight photospheric magnetic field, we have determined that the EBDs observed in the Hi-C FOV are located at the footpoints of the large-scale magnetic loops connecting AR 11520 to a decaying active region in the northern hemisphere. These properties suggest that EBDs might be a signature of an impulsive release of energy located at the base of coronal loops.

In terms of energetics, the magnetic energy estimate is of the order of $10^{26} \mathrm{erg}$, which is 100 times more than required by the nanoflare model suggested by Parker (1988). However, our estimate is an upper bound for the free magnetic energy that can be released, thus suggesting that smaller scale structures can exist. Nevertheless, Testa et al. (2013) and Winebarger et al. (2013) have estimated the radiated energy to $10^{24} \mathrm{erg}$, which is compatible with the order of magnitude of the magnetic energy; according to Emslie et al. (2012), who performed a complete study of the energy partition in eruptive events, the magnetic energy budget can be two orders of magnitude larger than the radiated energy.

Even if it is not possible to quantitatively compare due to the lack of spectroscopic data overlying the Hi-C FOV, it is important to mention the observations of Hara (2009), which showed that small-scale brightenings at the footpoints of coronal loops can be observed with a strong redshift velocity close to the local sound speed. Hara (2009) suggested that spatially unresolved $(<720 \mathrm{~km})$ structures could exist at the footpoint of coronal loops.

According to Walsh et al. (1995), the conduction timescale can be expressed as $\tau_{c}=p L^{2} / \kappa_{0} T^{7 / 2}$, where $p$ is the plasma pressure, $L$ is the characteristic length, $\kappa_{0}$ is the thermal conductivity in the corona $\left(\kappa_{0}=10^{-11}\right)$, and $T$ is the temperature. The characteristic length $L$ of the loop is chosen to be the loop length along which the heating is assumed to take place: $L=5 \mathrm{Mm}$. For a plasma pressure of 0.1 dyne $\mathrm{cm}^{-2}$ and a temperature range from 0.5 to $1.5 \mathrm{MK}$, the conductive timescale is between $6 \mathrm{~s}$ at $1.5 \mathrm{MK}$ and $5 \mathrm{~min}$ at $0.5 \mathrm{MK}$. A characteristic time of $25 \mathrm{~s}$ gives a characteristic temperature of $1 \mathrm{MK}$. Thus, the observed lifetime of EBDs may be compatible with the properties of a coronal plasma.

With the restricted observations of the $\mathrm{Hi}-\mathrm{C}$ instrument (short flight and limited FOV), questions arise for further study of these elementary events: how often and how common are EBDs in active regions? Are they always located at the footpoints of long coronal loops? What is their contribution toward the global heating of the corona? Can EBDs be associated with Type 2 spicules or even Ellerman bombs, as both have similar time scales? Several of these questions can be addressed by finding criteria that will enable the detection of EBDs in SDO/AIA images going forward, and by using high resolution chromospheric spectroscopic observations from IRIS.

We thank Jonathan Cirtain and Amy Winebarger for their help and discussion on the Hi-C instrument and to improve this manuscript. MSFC/NASA led the mission and partners include the Smithsonian Astrophysical Observatory in Cambridge, MA; Lockheed Martin's Solar Astrophysical Laboratory in Palo Alto, CA; the University of Central Lancashire in Lancashire, England; and the Lebedev Physical Institute of the Russian Academy of Sciences in Moscow.

\section{REFERENCES}

Alexander, C. E., Walsh, R. W., Régnier, S., et al. 2013, ApJL, 775, L32 Aly, J. J. 1984, ApJ, 283, 349

Amari, T., Luciani, J. F., Mikic, Z., \& Linker, J. 2000, ApJL, 529, L49

Beck, C., Bellot Rubio, L. R., Schlichenmaier, R., \& Sütterlin, P. 2007, A\&A, 472, 607

Berger, T. E., Rouppe van der Voort, L. H. M., Löfdahl, M. G., et al. 2004, A\&A, 428, 613

Boerner, P., Edwards, C., Lemen, J., et al. 2012, SoPh, 275, 41

Cirtain, J. W., Golub, L., Winebarger, A. R., et al. 2013, Natur, 493, 501

del Zanna, G., Landini, M., \& Mason, H. E. 2002, A\&A, 385, 968

Emslie, A. G., Dennis, B. R., Shih, A. Y., et al. 2012, ApJ, 759, 71

Guennou, C., Auchère, F., Soubrié, E., et al. 2012, ApJS, 203, 26

Hara, H. 2009, in ASP Conf. Ser. 415, The Second Hinode Science Meeting: Beyond Discovery-Toward Understanding, ed. B. Lites, M. Cheung, T. Magara, J. Mariska, \& K. Reeves (San Francisco, CA: ASP), 252 
Jordan, C., Ayres, T. R., Brown, A., Linsky, J. L., \& Simon, T. 1987, MNRAS 225,903

Klimchuk, J. A. 2006, SoPh, 234, 41

Kobayashi, K., Cirtain, J., Winebarger, A. R., et al. 2013, submitted to SoPh

Lemen, J. R., Title, A. M., Akin, D. J., et al. 2012, SoPh, 275, 17

Mackay, D. H., Galsgaard, K., Priest, E. R., \& Foley, C. R. 2000, SoPh, 193, 93

Parker, E. N. 1988, ApJ, 330, 474

Pesnell, W. D., Thompson, B. J., \& Chamberlin, P. C. 2012, SoPh, 275, 3

Priest, E. R., Foley, C. R., Heyvaerts, J., et al. 2000, ApJ, 539, 1002

Reale, F. 2002, ApJ, 580, 566

Reale, F. 2010, LRSP, 7, 5

Régnier, S., \& Priest, E. R. 2007, A\&A, 468, 701
Rouppe van der Voort, L. H. M., Hansteen, V. H., Carlsson, M., et al. 2005, A\&A, 435,327

Rutten, R. J., Hammerschlag, R. H., Bettonvil, F. C. M., Sütterlin, P., \& de Wijn, A. G. 2004, A\&A, 413, 1183

Scharmer, G. B., Bjelksjo, K., Korhonen, T. K., Lindberg, B., \& Petterson, B. 2003, Proc. SPIE, 4853, 341

Scherrer, P. H., Schou, J., Bush, R. I., et al. 2012, SoPh, 275, 207

Testa, P., De Pontieu, B., Martínez-Sykora, J., et al. 2013, ApJL, 770, L1

Viall, N. M., \& Klimchuk, J. A. 2011, ApJ, 738, 24

Viall, N. M., \& Klimchuk, J. A. 2012, ApJ, 753, 35

Walsh, R. W., Bell, G. E., \& Hood, A. W. 1995, SoPh, 161, 83

Winebarger, A. R., Walsh, R. W., Moore, R., et al. 2013, ApJ, 771, 21

Winebarger, A. R., Warren, H. P., \& Seaton, D. B. 2003, ApJ, 593, 1164 